\title{
QUANTIDADE E QUALIDADE: DUAS ABORDAGENS DA MEMÓRIA VISUO-ESPACIAL.
}

\author{
César Alexis Galera ${ }^{l}$ \\ Universidade de São Paulo - Ribeirão Preto \\ Susi Lippi Marques Oliveira \\ Universidade Federal de São Carlos
}

\begin{abstract}
Resumo: Neste texto apresentamos alguns aspectos que tem caracterizado diferentes abordagens no estudo da memória a curto prazo. O enfoque quantitativo, proposto por Ebbinghaus (1885), aborda a memória em termos de sua capacidade de armazenamento. A memória é vista como um armazém no qual a informação é depositada e posteriormente recuperada; sua capacidade é determinada tanto em termos do número de itens que consegue armazenar, como em termos da duração dos itens armazenados. O enfoque qualitativo, com bases na tradição da Gestalt e no trabalho de Bartlett (1932), aborda a memória como um processo de representação ou de reconstrução de eventos passados. Nesta abordagem a memória é avaliada em termos da correspondência entre a representação e aquilo que é representado.
\end{abstract}

Palavras-chave: Memória a curto prazo, memória visuo-espacial.

\section{QUANTITY AND QUALITY: TWO APPROACHES TO VISUOSPATIAL MEMORY.}

Abstract: In this text we present some aspects that have characterized different approaches to the study of the short-term memory. The quantitative approach, considered by Ebbinghaus (1985), considers the memory in terms of its capacity of storage. The memory is seen as a warehouse in which the information is deposited and later retrieved; its capacity is determined by the number of items that can be stored and in terms of the duration of the stored items. The qualitative approach, based in the Gestalt tradition and on Bartlett (1932), views the memory as a process of representation or reconstruction of past events. In this approach the memory is evaluated in terms of the correspondence between the representation and what it is represented

Key-words: Short-term memory, visuo-spatial memory.

Há alguns anos estávamos dando uma festa em casa quando o telefone tocou. Era um dos convidados, um velho amigo, que havia sofrido um acidente ao sair de casa. Para nossa sorte o acidente não havia sido muito sério. Ele estava saindo de seu apartamento, quando, previdente, pensou que seria perigoso entrar na casa, às escuras, depois de algumas cervejas. E voltou para acender a luz. O que poderia ter acontecido na volta, aconteceu na ida. Meu amigo tropeçou numa mesa de centro, caiu, trincou um osso do braço e quebrou o nariz. O acidente, é claro, foi muito desagradável, mas o que nos cha-

Artigo recebido para publicação em 11/09/2003; aceito em 15/12/2003. Endereço para correspondência: César Alexis Galera - Faculdade de Filosofia, Ciências e Letras de Ribeirão Preto - USP - Departamento de Psicologia e Educação - Av. Bandeirantes, 3900 - CEP. 14040-901, Monte Alegre, Ribeirão Preto, SP, Brasil - E-mail: algalera@usp.br mou mais a atenção foi que a mesa de centro estava no mesmo lugar há mais de 20 anos.

Esse acidente nos fez pensar, em primeiro lugar que as mesas de centro são perigosas, e em segundo, que existe um descompasso entre nossa representação do ambiente e o próprio ambiente. Isso é ainda mais surpreendente quando a nossa sensação é que lembramos perfeitamente, nos mínimos detalhes, dos ambientes em que vivemos. É surpreendente que possamos ter imagens nítidas e robustas de nossa sala de estar, e que estas imagens não nos permitam, na ausência da luz, interagir adequadamente com esse ambiente. Neste texto, em homenagem a um outro velho amigo, vamos apresentar algumas idéias sobre as representações visuo-espaciais para a cognição humana. 
A tradição iniciada por Ebbinghaus, de abordar a memória como um armazenador cujas propriedades podem ser expressas por taxas de esquecimento, tem dominado os estudos da memória, principalmente da memória a curto prazo. Os estudos sobre a capacidade desse sistema de memória (Miller, 1956), da duração da informação memorizada (Brown, 1958; Peterson \& Peterson, 1959), assim como a maioria dos estudos realizados nos últimos 50 anos tem considerado a memória como um armazém no qual dão entrada informações sobre objetos específicos que serão recuperadas em algum momento do futuro. Esse sistema armazenador é avaliado em termos da quantidade de informação que pode ser recuperada depois de um certo intervalo de tempo.

Uma abordagem muito diferente daquela iniciada por Ebbinghaus, presente nos estudos de memória visual realizados no século XIX, adotada pelos psicólogos da Gestalt (Koffka, 1935) e também pelos psicólogos ingleses (Bartlett, 1932) considerava a memória como um processo de elaboração, um processo ativo a ser avaliado tanto em termos qualitativos como em termos de sua precisão, em termos de sua fidelidade à informação representada. De acordo com esta concepção, a natureza do erro, e não sua freqüência, pode revelar com mais profundidade a natureza da memória humana.

Neste estudo vamos apresentar algumas conquistas conseguidas por essas duas abordagens no estudo da memória visuo-espacial a curto prazo.

\section{Memória como depósito de capacidade limitada}

Na década de 60 os estudos realizados segundo a perspectiva clássica, voltados à duração e à capacidade de armazenagem do sistema de memória a curto prazo culminaram no modelo unitário de Atkinson e Shiffrin (1968). Neste modelo a memória a curto prazo seria um sistema simples, unitário, responsável por manter a informação recebida do ambiente durante o tempo necessário para transferila para a memória a longo prazo. Essa concepção, da memória a curto prazo como um sistema unitário foi abalada por estudos experimentais e por casos da clínica neurofisiológica. Resultados experimentais importantes para uma mudança na compreensão da memória a curto prazo foram encontrados por Alan Baddeley em estudos em que os sujeitos deveriam realizar duas tarefas simultaneamente. Esses estudos mostraram que os sujeitos eram realmente capazes de realizar as duas tarefas com uma precisão acima do que poderia se esperar com base em um modelo de armazenamento único. Atualmente o modelo de memória operacional (working memory) desenvolvido originalmente por Baddeley e Hitch (1974) envolve quatro subsistemas funcionais. A informação verbal e auditiva é armazenada por um laço fonológico. Um rascunho visuo-espacial tem a função de manter e manipular a informação referente aos objetos e às relações espaciais entre eles. Um buffer episódico tem a função de armazenar temporariamente a informação multimodal, aglutinando a informação dos outros subsistemas e da memória a longo prazo numa representação episódica unitária. O fluxo de informação vinda do ambiente e da memória a longo prazo para os subsistemas da memória operacional está sob a supervisão de um sistema executivo central. Juntos esses subsistemas estariam envolvidos em atividades cognitivas superiores tais como o processamento da linguagem, leitura, solução de problemas e na produção da própria consciência (Baddeley, 1986, 2000).

As características do laço fonológico estão bem estabelecidas, mas se conhece relativamente menos sobre o funcionamento do sistema de memória visuo-espacial a curto prazo. Existem evidências consistentes de que este subsistema funciona de forma independente do sistema fonológico. Por exemplo, o desempenho numa tarefa que exige a recordação de material visual é fortemente perturbado pela execução simultânea de uma tarefa de rastreamento visuoespacial, mas a tarefa de rastreamento não interfere no desempenho de uma tarefa que exige a recordação de material verbal (Baddeley, Grant, Wight, \& Thompson, 1975). Ou seja, parece haver alguma sobreposição entre os recursos cognitivos necessários para manter o material visual e os recursos necessários para realizar a tarefa de rastreamento (mas não entre os recursos necessários ao rastreamento e à recordação do material verbal). Por outro lado, resultados equivalentes obtidos com uma tarefa de rastreamento realizada com feedback sonoro, sugerem que a tarefa de rastreamento envolve um sistema de processamento espacial, não visual, independente do sistema fonológico (Baddeley, 1986). 
Evidências de sistema de memória visual a curto prazo, independente do laço fonológico foram apresentadas por Phillips e Christtie (1977a, b) em um estudo com uma tarefa de memorização de estímulos visuais sem nome. Estes autores determinaram a capacidade da memória visual a curto prazo utilizando estímulos produzidos pelo preenchimento aleatório de oito caselas numa matriz 4 x 4. Uma lista desses estímulos era apresentada para memorização; logo em seguida a lista era apresentada para julgamento, mas em ordem inversa àquela apresentada originalmente. Em cada prova o sujeito deveria decidir se os estímulos eram iguais nas duas listas. Os resultados mostram um forte efeito de recência restrito ao último estímulo apresentado, isto é, apenas o último padrão da lista era reconhecido em quase $100 \%$ das provas, enquanto a porcentagem de reconhecimento dos outros estímulos girava em torno de $60 \%$. A introdução de uma tarefa aritmética, a soma de cinco dígitos realizada durante o intervalo de retenção, ou contar de trás para frente de três em três, eliminou completamente o efeito de recência, mas não afetou a taxa de reconhecimento dos primeiros estímulos da lista (Phillips \& Christtie, 1977b). Parece evidente que os sujeitos conseguiam manter apenas o último estímulo "na mente", até que fosse testado, mas não podiam fazer o mesmo com os outros estímulos. O efeito seletivo da tarefa aritmética sugere fortemente, segundo os autores, que recursos atencionais externos ao sistema de memória visual são necessários à manutenção do último estímulo da lista. Quando esses recursos são drenados pela tarefa aritmética o sistema não é mais capaz de manter o último estímulo ativo.

Uma possível independência entre os componentes visuais e espaciais do sistema de memória visuo-espacial foi sugerida por Logie (1986) num estudo em que a tarefa de Brooks (1967) foi realizada simultaneamente com outras tarefas. Na tarefa de Brooks, o sujeito é instruído a imaginar uma matriz de 4 x 4 , em cujas caselas ele deve colocar números. A tarefa pode tanto favorecer a utilização de imagem visual, como pode exigir a memória verbal. Os resultados obtidos por Logie mostram que a apresentação de estímulos irrelevantes visuais interfere fortemente no desempenho quando o sujeito utiliza uma estratégia visual, mas nesse caso o desempenho não é afetado pela apresentação de estímulos irrelevantes verbais. Por outro lado, quando a estratégia de memorização é verbal o desempenho é fortemente prejudicado pela apresentação de estímulos irrelevantes verbais, mas não pela apresentação de estímulos irrelevantes visuais. Estes resultados sugerem, de acordo com o autor, que o sistema visuoespacial poderia ser melhor compreendido se fosse subdividido em dois componentes, um voltado ao processamento da informação espacial e outro à informação visual. Tais subsistemas, além de estarem mais ajustados aos resultados experimentais, também seriam consistentes com as evidências neurofisiológicas de sistemas separados dedicados ao processamento do "quê" e do "onde" (Ungerleider \& Mishkin, 1982; Milner \& Goodale, 1995).

De acordo com Logie (1995) um sistema de memória a curto prazo deve ser capaz de receber e armazenar informações vindas do ambiente e da memória a longo prazo. Uma vez que essa informação é mantida num armazenador temporário, do qual pode ser deslocada, seja pelo decaimento do traço de memória, seja pelo deslocamento provocado por novas informações que chegam ao sistema, o sistema deve ter meios de estender o período de retenção, se isto for necessário. Finalmente, um sistema de memória que tem por função armazenar e manipular a informação visual e espacial deve codificar essa informação de forma a manter as características visuais e espaciais com as quais o sistema deve operar. No entanto, a abordagem clássica da memória, da qual Logie é um dos expoentes, tem se preocupado quase que exclusivamente com a capacidade do armazenamento de listas de estímulos e com os mecanismos necessários para manter a informação memorizada, mas tem dado pouca atenção à acurácia com que a informação ambiental é representada. Raramente esta abordagem tem se preocupado com a fidedignidade, com a correspondência entre a representação e o representado.

\section{Memória como percepção do passado}

A abordagem qualitativa da memória, como representação, como reconstrução da experiência passada, remonta à Psicologia da Gestalt e aos estudos realizados por Sir Frederic Bartlett, entre outros. 
Nesta abordagem a memória é avaliada nos termos de sua correspondência com eventos passados, e não em termos número de itens memorizados que podem ser recuperados. Em seu estudo com a lenda da Guerra dos Espíritos, uma lenda de índios norte-americanos, Bartlett (1932) mostrou que os relatos obtidos com estudantes de Oxford eram incompletos, distorcidos e influenciados por fatores pessoais e sociais. A recordação, de acordo com Bartlett, é guiada por schemas, estruturas mentais determinadas culturalmente, que oferecem um cenário cognitivo no qual a informação nova é integrada. A informação nova é codificada em termos das informações existentes no schema. E, quando deve ser recuperada, traz consigo informações mais antigas, existentes no schema no momento em que foi codificada.

Estudos mais recentes têm sugerido que a recordação é afetada por três tipos principais de omissões e distorções: a seleção, a interpretação e a integração (Alba \& Hasher, 1983). O schema ativo no momento em que ocorre um evento determina que aspectos daquele evento serão selecionados e memorizados. O schema ativo no momento em que ocorre um evento fornece o conhecimento necessário para que possamos interpretar o evento. Finalmente, a combinação, a integração de novas e velhas informações será outra fonte importante de distorções da informação memorizada (Baguley \& Payne, 2000).

Um pouco antes dos estudos de Bartlett, e muito antes dos estudos de Alba e Hasher (1983), os psicólogos da Gestalt mostraram que a recordação de cenas e desenhos também é um processo ativo, com princípios equivalentes àqueles que regem a percepção visual. De acordo com Wulf (1922; citado em Woodworth, 1938) dois tipos principais de mudanças regem a produção de desenhos a partir de memória, um que poderia ser entendido como “desbastamento", que envolve a ampliação de algumas características do desenho original, e o "amaciamento" de outras características. Esses tipos de mudanças seriam determinados por três fatores: a normalização, o apontamento e a tendência à boa forma. A reprodução de desenhos feita de memória tende a simplificá-los. A reprodução de um desenho traz apenas seus traços mais relevantes, esses traços são interpretados em função de sua organização e combinados com as informações compatíveis com a interpretação (Woodworth, 1938).

De acordo com Koriat, Goldsmith e Pansky (2000) a abordagem da memória em termos de correspondência com eventos passados pode ser diferenciada da abordagem clássica em vários atributos. A memória é vista como uma representação de um evento passado; os relatos de memória são tratados como descrições, como proposições que tem um valor de verdade, e não como uma lista de itens recuperados. Nesta abordagem se focaliza a acurácia do relato; a memória é avaliada em termos de sua concordância com a realidade, em termos de sua exatidão. O esquecimento é visto como uma perda de correspondência entre o relato e o evento real, como uma distorção, e não simplesmente como o decaimento ou como o resultado da interferência entre traços. Esta forma de compreender o esquecimento enfatiza a importância da análise qualitativa das distorções introduzidas no relato. A abordagem da memória em termos de correspondência tem muito a ver com a forma como pensamos a percepção. Nos estudos da percepção procuramos estabelecer a correspondência entre o psíquico e o físico, e também compreender como, nas ilusões, nossa percepção se afasta da realidade. Da mesma forma, a memória pode ser vista como uma percepção do passado, então a questão se torna: em que medida esta percepção é verídica ou ilusória? (Koriat, Goldsmith, \& Pansky, 2000).

Esta afinidade entre memória e percepção explicitada na abordagem da correspondência oferece um arcabouço teórico para o estudo da psicofísica da memória. A psicofísica da memória compreende a memória como percepção do passado e procura estabelecer as relações funcionais entre a magnitude física e a magnitude psicológica dos estímulos quando estes são julgados a partir de representações memorizadas (Algom, 1992). Este tipo de estudo adota os mesmos métodos quantitativos da psicofísica perceptiva para estabelecer o grau em que a informação espacial é preservada na memória. A aplicação de métodos de estimação de magnitudes em situações de julgamento perceptivo e em situações de julgamento de memória revela que os expoentes da função potência que relaciona a magnitude psicológica à magnitude física (Stevens, 1975) são menores na situação de julgamentos de memória do que 
na condição perceptiva.

Para alguns autores o rebaixamento do expoente obtido através de julgamentos feitos de memória tem sido atribuído ao aumento da incerteza que os observadores experimentam quando devem fazer julgamentos baseados em representações memorizadas (Moyer, Sklarew, \& Whiting, 1982; Algom, Wolf, \& Bergman, 1985; Radvansky, Carlson-Radvansky, \& Irwin, 1995; Marques, 1996). Mas este rebaixamento do expoente também tem sido explicado pela hipótese da repercepção. De acordo com esta explicação, a codificação inicial do estímulo (transdução) obedece a uma função de potência; o julgamento de memória, realizado sobre a representação codificada do estímulo, também obedece a uma função de potência. Dessa forma, supondo que as duas transformações, a perceptiva e a mnemônica, são idênticas, os expoentes dos julgamentos de memória deveriam tender ao quadrado dos expoentes obtidos com julgamentos perceptivos, mas isso nem sempre ocorre (Da Silva, Fukusima, Dalamangas, Marques, \& Ruiz, 1988; Kerst \& Howard, 1978).

Ao considerar que o processo de "transdução mnemônica” é semelhante ao processo de transdução sensorial, os proponentes da hipótese reperceptiva adotam a concepção da memória como a percepção do passado, e devem também admitir que as representações utilizadas nos julgamentos de memória são equivalentes às representações utilizadas nos julgamentos preceptivos. De fato existem evidencias de que muitas das estruturas do córtex envolvidas na percepção, no processamento primário, são as mesmas envolvidas nas imagens mentais (Farah, 1985, 1989, 1999). Também existem evidências de que o processo de julgamento exigido nos experimentos de percepção é muito semelhante aos processos exigidos nos julgamentos baseados em imagens mentais (Hubbard \& Stoeckig, 1992; Kosslyn, Ball, \& Reisser, 1978; Kerst \& Howard, 1978, Baird \& Harder, 2000).

Nós investigamos a natureza da representação memorizada das relações espaciais entre objetos de uma cena em tarefas de julgamentos de magnitude e produção de desenhos através da introdução de tarefas intervenientes. A tarefa interveniente é uma das técnicas mais utilizadas nos estudos da memó- ria. A idéia básica é que o sistema cognitivo é limitado para armazenar e processar a informação a curto prazo. Os conteúdos armazenados estão sujeitos a decair com o tempo, a menos que sejam recitados por um processo de recitação visual. A tarefa interveniente tem exatamente o papel de impedir que a informação visual seja recitada durante o intervalo de retenção. Além disso, se considerarmos que cada tipo de representação armazenada utiliza recursos específicos, verbais ou visuo-espaciais, devemos esperar um efeito maior de uma tarefa interveniente que utilize os mesmos recursos que uma representação armazenada. Por exemplo, o desempenho em uma tarefa verbal será mais prejudicado por uma tarefa interveniente verbal do que por uma tarefa interveniente visuo-espacial, e vice-versa (Baddeley \& Hitch, 1974; Bower \& Clapper, 1993; Logie, 1995).

Nossos resultados mostram que os julgamentos de memória são bastante afetados pela realização de tarefas intervenientes visuo-espacial e aritmética no intervalo de retenção, entre o estudo de uma cena e o momento em que devem ser feitos os julgamentos e o desenho. A tarefa aritmética também provoca uma diminuição nos expoentes obtidos com julgamento de magnitude e com desenho, e a tarefa visuo-espacial provoca um aumento no expoente obtido com o julgamento mas não afeta o desenho. Seria lógico pensar que ambas as tarefas psicofísicas, de estimação de magnitude e produção de desenho, utilizam representações semelhantes. Esta suposição é plausível, mas deve-se considerar que as tarefas apresentam exigências diferentes. Por exemplo, na tarefa de estimação de magnitude o sujeito não precisa lembrar o nome dos objetos, esta informação é fornecida pela própria situação experimental, no caderno onde o sujeito deve realizar seus julgamentos. Por outro lado, na produção de desenho, o sujeito não dispõe dessa informação. Nesta situação o sujeito deve lembrar as distâncias, e também os objetos que estavam presentes na cena. Essa recordação dos objetos da cena pode estar utilizando o laço fonológico. Enquanto que na tarefa de estimação de magnitude o sujeito não precisa recordar os objetos, essa informação é dada pelo experimentador. Essa diferença no procedimento pode explicar a interferência da tarefa verbal-arit- 
mética na produção de desenho, e a ausência de interferência dessa tarefa na estimação de magnitude. No entanto, a pequena diminuição, não significativa, provocada pela tarefa interveniente visuo-espacial na produção de desenho, permanece por ser explicada (Marques, Galera, \& Eik, 2000).

Então, o que as tarefas intervenientes podem nos dizer a respeito da controvérsia entre repercepção e tendência central de julgamentos? A hipótese de repercepção considera que existe uma concordância entre as representações utilizadas nos julgamentos perceptivos e nos julgamentos de memória. Neste sentido, a interferência produzida pela tarefa interveniente visuo-espacial é mais uma evidência que fortalece a suposição de que os julgamentos de memória são feitos com base em representações que guardam as características visuo-espaciais da cena. Mas, deve-se também levar em conta que a manutenção das representações espaciais também depende da informação verbal proporcionada pelo nome dos objetos presentes na cena. A interferência provocada pela tarefa verbal-aritmética, diminuindo o expoente obtido através do desenho, sugere que a recitação verbal, impedida pela tarefa interveniente, é um componente importante, não considerado, nem pela hipótese reperceptiva, nem pela hipótese da tendência central. Atualmente, novos estudos estão sendo conduzidos visando uma melhor compreensão dos pontos abordados neste trabalho.

\section{Conclusão}

Nós começamos este texto falando sobre um acidente ocorrido em um ambiente natural, muito conhecido, e conhecido há muito tempo, e acabamos falando apenas de estudos em situações bastante artificiais e pouco conhecidas. O que esses estudos nos contam sobre a dificuldade em caminhar no escuro, num ambiente que devíamos conhecer tão bem quanto a palma de nossas mão? Pouco, muito pouco. Nós temos uma memória visual a curto prazo de capacidade bastante limitada, com uma amplitude variando de 1 a 3 itens e que parece depender de recursos atencionais (Philips, 1983; D.E. Broadbent \& M.H.P. Broadbent, 1981). Não podemos manter em nossa mente mais do que um ou outro estímulo visual, e mesmo isso nos exige um certo esforço atencional que prejudica nossa capacidade de fazer outras coisas simultaneamente (Oliveira \& Galera, $\mathrm{s} / \mathrm{d})$.

Navegar por um ambiente bem iluminado, sem esbarrar nos móveis e objetos, envolve um cálculo complexo de uma infinidade de fatores, entre eles a velocidade, direção e atualização da nossa própria posição espacial, tudo isso realizado em tempo real. Quando nos movemos por uma sala bem iluminada nossa visão cumpre dois papéis, um deles é o de coordenação visuo-motora, que nos permite navegar entre os objetos, o outro está relacionado à nossa habilidade para reconhecer os objetos. De acordo com Milner e Goodale (1995), as áreas do córtex visual dos primatas podem ser divididas em pelo menos dois agrupamentos neurais funcionalmente diferentes. Um dos agrupamentos (sistema “onde”) está ligado ao controle do movimento, o outro (sistema "quê") está associado aos sistemas de memória, planejamento e a outros processos cognitivos. No escuro, o sistema "onde" é inútil, e somos obrigados a utilizar nossas lembranças, nossas representações, que embora sejam úteis para o reconhecimento dos objetos, não são exatamente adequados para o deslocamento no espaço. As conseqüências são conhecidas por aqueles que tentam usar uma ferramenta para um fim que não lhe é próprio.

Além disso, parece existir outra razão pela qual não devemos (ou não podemos) utilizar nossa memória visual para guiar nosso deslocamento no espaço. E esse motivo diz respeito exatamente ao problema da correspondência entre representação e ambiente. Nós costumamos ter uma memória vívida, nítida e clara dos ambientes onde vivemos, dos objetos que utilizamos todos os dias, mas raramente nos perguntamos se essa sensação de nitidez, se essa clareza, corresponde à realidade. Se perguntássemos seríamos obrigados a responder que nossa memória a longo prazo é muito pior do que gostaríamos de admitir. Nickerson e Adams (1979) mostraram nossa falta de memória a longo prazo num estudo em que pediram a adultos americanos para desenharem e identificarem os traços presentes na moeda de um centavo de dólar. Outro estudo mais recente mostra que nossa memória para os teclados com os quais convivemos diariamente não é muito melhor (Rinck, 1999). Os resultados desses estudos mostram que a 
convivência com um objeto e a habilidade para reconhecê-lo não garante que esse objeto é representado acuradamente na memória, pelo contrário, sugerem que memória visual a longo prazo é muito menos elaborada do que nós freqüentemente supomos e gostamos de acreditar.

\section{Referências Bibliográficas}

Alba, J.W., \& Hasher, L. (1983). Is memory schematic? Psychological Bulletin, 93, 203-231.

Algom, D. (1992). Memory psychophysics: an examination of its perceptual and cognitive prospects. Em D. Algom (Org.), Psychophysical approaches to cognition (pp.441-513). Amsterdam: Elsevier.

Algom, D., Wolf, Y., \& Bergman, B. (1985). Integration of stimulus dimensions in perception on memory: composition rules and psychophysical relations. Journal of Experimental Psychology: General, 114, 451-471.

Atkinson, R.C., \& Shiffrin, R.M. (1968). Human memory: A proposed system and its control processes. Em K.W. Spence \& J.T. Spence (Orgs.), The psychology of learning and motivation: advances in research and theory, Vol. 2 (pp. 89195). New York: Academic Press.

Baddeley, A. (1986). Working memory. New York: Oxford University. Press.

Baddeley, A. (2000). The episodic buffer: a new component of working memory? Trends in Cognitive Sciences, 4, 419-423.

Baddeley, A., Grant, W., Wight, E., \& Thompson, N. (1975). Imagery and visual working memory. Em S. Dornic (Org.), Attention and performance, Vol. V (pp. 205-217), London, Academic Press.

Baddeley, A., \& Hitch, G.J. (1974). Working Memory. Em G. Bower (Org.), The psychology of learning and motivation, Vol. 8. New York: Academic Press.

Baird, J.C., \& Harder, K.A. (2000). The Psychophysics of imagery. Perception \& Psychophysics, 62, 113-126.
Baguley, T., \& Payne, S.J. (2000). Long-term memory for spatial and temporal mental models includes construction processes and model structure. Quarterly Journal of Experimental Psychology: Human Experimental Psychology, 53A, 479-512.

Bartlett, F.C. (1932). Remembering: a study in experimental and social psychology. New York: Academic Press.

Bower, G.H., \& Clapper, J.P. (1993). Experimental Methods in Cognitive Science. Em M.I. Posner (Org.), Foundations of Cognitive Science. Cambridge: MIT Press.

Broadbent, D.E., \& Broadbent, M.H.P. (1981). Recency effects in visual memory. Quarterly Journal of Experimental Psychology, 33A, 1-15.

Brooks, L.R. (1967). The suppression of visualization by reading. Quarterly Journal of Experimental Psychology, 19, 289-299

Brown, J. (1958). Some tests of the decay theory of immediate memory. Quarterly Journal of Experimental Psychology, 10, 12-21

Da Silva, J.A., Fukusima, S.S., Dalamangas, A.M., Marques, S.L., \& Ruiz, E.M. (1988). Psicofísica da Memória. Arquivos Brasileiros de Psicologia, 40(3), 3-41.

Ebbinghaus, H. (1885). Memory: a contribution to Experimental Psychology (tradução H.A. Ruger, \& C.E. Bussenues, 1913). New York: Teachers College/Columbia University.

Farah, M. (1985). Psychophysical evidence for a shared representational medium for mental images and percepts. Journal of Experimental Psychology: General, 114, 91-103.

Farah, M. (1989). The neural basis of mental imagery. Trends in Neurosciences, 12, 395-399.

Farah, M. (1999). The neural basis of mental imagery. Em M.S. Gazzaniga (Org.), The Cognitive Neurosciences. Cambridge: MIT Press.

Hubbard, T.L., \& Stoeckig, K. (1992). The representation of pitch in musical images. Em D. Reisberg (Org.), Auditory imagery. Hillsdale: Album. 
Kerst, S.M., \& Howard, J.H. (1978). Memory Psychophysics for Visual Area and Length. Memory and Cognition, 6, 237-335.

Koffka, K. (1935). Principles of Gestalt Psychology. New York: Harcourt Brace.

Koriat, A, Goldsmith, M., \& Pansky (2000). Toward a psychology of memory accuracy. Annual Review of Psychology, 51, 481-537.

Kosslyn, S.M., Ball. T.M., \& Reisser, B.J. (1978). Visual images preserve metric of spatial information: evidence from studies of image scanning. Journal of Experimental Psychology: Human Perception and Performance, 4, 47-60.

Logie, R.H. (1986). Visuo-spatial processing in working memory. Quarterly Journal of Experimental Psychology, 38A, 229-247.

Logie, R.H. (1995). Visuo-spatial working memory. London: Lawrence Erlbaum Associates.

Marques, S.L. (1996). Funções perceptivas e mnemônicas de área e volume familiares e não familiares sob instruções aparente e objetiva. Tese de Doutorado. Universidade de São Paulo - Ribeirão Preto, SP..

Marques, S.L., Galera, C., \& Eik, G. (2000). Efeito de tarefas intervenientes verbais e visuo-espaciais sobre as estimativas mnemônicas em diferentes procedimentos psicofísicos. Arquivos Brasileiros de Psicologia, 52, 26-34

Miller, G.A. (1956). The magical number seven, plus or minus two: some limits on our capacity for processing information. Psychological Review, 63, 81-97.

Milner, D.A., \& Goodale, M.A. (1995). The visual brain in action. Oxford: Oxford University Press.

Moyer, R.S., Sklarew, P., \& Whiting, J.C. (1982). Memory Psychophysics. Em H.G. Gussler, \& H. Petzold (Orgs.). Psychophysical Judgments and the Process of Perception (pp. 35-46). Berlim: VEB Deutscher Verlag der Wissenchaften.

Nickerson, R. S. \& Adams, M. J. (1979). Long-term memory for common object. Cognitive Psychology, 11, 287-307.
Oliveira, R.G., \& Galera, C. (s/d). A memória visuoespacial a curto prazo utiliza recursos atencionais gerais? Manuscrito em preparo.

Peterson, L.R., \& Peterson, M. (1959). Short-term retention of individual items. Journal of Experimental Psychology, 58, 193-198.

Phillips, W.A., \& Christie, D.F.M. (1977a). Components of visual memory. Quarterly Journal of Experimental Psychology, 29, 117133.

Phillips, W.A., \& Christie, D.F.M. (1977b). Interference with visualization. Quarterly Journal of Experimental Psychology, 29, 637650.

Phillips, W.A. (1983). Short-term visual memory. Philosophical Transaction of the Royal Society, 302, 295-309.

Radvansky, G.A., Carlson-Radvansky, L.A., \& Irwin, D.E. (1995). Uncertainty in estimating distances from memory. Memory \& Cognition, 23(5), 596606.

Rinck, M. (1999). Memory for everyday objects: where are the digits on numerical keypads? Applied Cognitive Psychology, 13, 329-350.

Stevens, S.S. (1975). Psychophysics: Introduction to its perceptual, neural and social prospects. New York: Wiley.

Ungerleider, L.G. \& Mishkin, M. (1982). Two cortical visual systems. Em D.J. Ingle, M.A. Goodale, \& R.J.W. Mansfield (Orgs.), Analysis of visual behavior (pp. 549-586). Cambridge: MIT Press.

Woodworth, R.S. (1938). Experimental Psychology. New York, Holt.

\section{Agradecimentos:}

Agradecemos ao CNPq (522624/95-2) e aos alunos do curso de graduação em Psicologia da UFSCar e da USP envolvidos neste projeto: Ivana Regina Panosso, Raquel Cordioli, Náira Verceze e Rodrigo Gonçalves. 